\title{
Subacute sclerosing panencephalitis in a Roma adolescent
}

$\underline{\text { M. Noni }}{ }^{1}$, N. Diamantopoulos ${ }^{2}$, V. Bellou ${ }^{2}$, M.-R. Pons ${ }^{1}$, V. Spoulou ${ }^{1}$

1 1st Department of Pediatrics, "Aghia Sophia” Children's Hospital, Athens, Greece 2 Pediatric Clinic, "Karamandanio" General Hospital, Patra, Greece

\section{ГBackground:}

Subacute sclerosing panencephalitis (SSPE) is a rare and fatal complication of measles characterized by cognitive and motor deterioration, with death typically joccurring within one to three years of symptom onset. Prior to measles vaccination programs, the incidence of SSPE in developed countries was "estimated at 1 case per 100,000 population, but was 100 times higher in some developing nations suggesting that environmental or genetic factors, or specific "measles genotypes might be risk factors. A consistent SSPE risk factor has been " measles infection before the second birthday.

-

\section{Case presentation: " - " - " - " - " " " " " " " - " - "}

:*Patient's history: 13-year-old boy presented with frequent myoclonus started a month ago leading to falls on the ground.

* Medical history: totally unvaccinated and infected with measles at the age of two years.

* Clinical examination: dysarthria, gait disorder, personality changes and "intellectual deterioration.

EEG: periodic bursts of high-amplitude, slow-wave complexes every 6 seconds :

correlated with myoclonous (Figure 1)

* Brain MRI: atypical findings on the left lobe (Figure 2).

*CSF analysis: elevated measles IgM and IgG antibody titters, elevated IgG index and presence of oligoclonal bands (Table).

* Treatment approach:

- $1^{\text {st }}$ step: valproic acid, levetiracetam and intrathecal interferon a

- $2^{\text {nd }}$ step: intraventricular infusions through an ommaya reservoir

- $3^{\text {rd }}$ step: isoprinoside

* Patient's follow-up:

- serious complications (convulsions, high fever, progressive weakness, rigidity) followed each intraventricular infusion

- temporary discontinuation of interferon a.

- without clinical deterioration after a 6-month follow-up period

Figure 1. Patient's EEG revealed a highly

characteristic pattern of SSPE
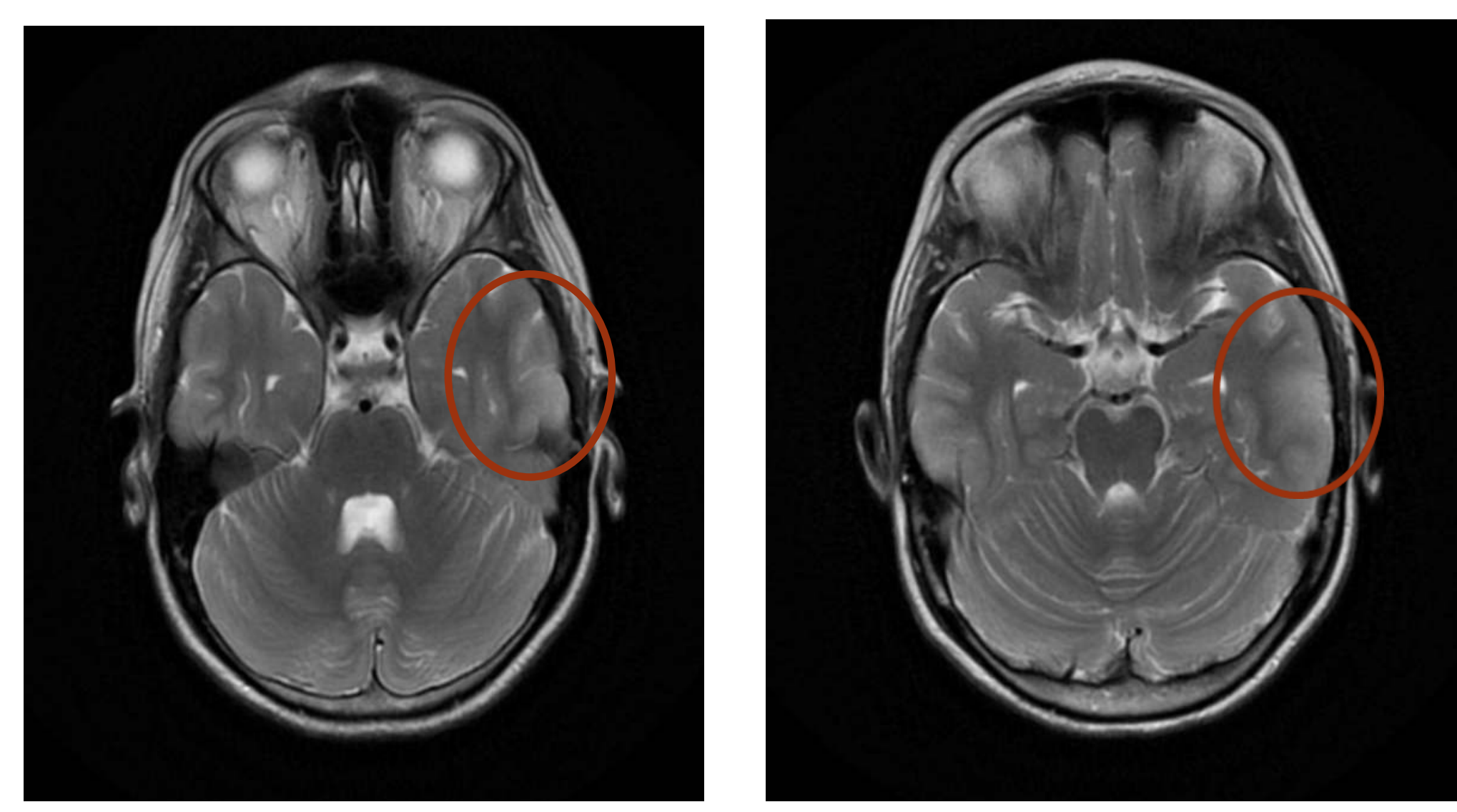

Figure 2. MRI T2w revealed increased signal on the left temporal lobe

\begin{tabular}{|l|l|}
\hline & \multicolumn{1}{|c|}{ Results } \\
\hline Abs IgG against measles on serum & $\mathbf{4 8 , 3} \mathrm{NTU}(\mathrm{NV}<9)$ \\
\hline Abs IgM against measles on serum & 0,02 (index) $(\mathrm{NV}<0,1)$ \\
\hline CSF & $\begin{array}{l}\text { cells }=3 \text {, protein }=38 \mathrm{mgr} / \mathrm{dL}, \\
\text { glucose }=61 \mathrm{mgr} / \mathrm{dL}\end{array}$ \\
\hline Abs IgG against measles on CSF & $\mathbf{3 4 , 8} \mathrm{NTU}(\mathrm{NV}<9)$ \\
\hline Abs IgM against measles on CSF & 0,01 (index) $(\mathrm{NV}<0,1)$ \\
\hline IgG index & $\mathbf{2 , 7 5}(\mathrm{NV}<0,66)$ \\
\hline Oligoclonal bands on CSF & positive (type 3) \\
\hline PCR on CSF sample & $\begin{array}{l}\text { measles: slightly positive } \\
\text { VZV, AdV, enterovirus, parvovirus } \\
\text { B19, } \mathrm{HSV} 1,2: \text { negative }\end{array}$ \\
\hline
\end{tabular}

Table. Serum and CSF analysis

\section{Learning Points/ Discussion:}

SSPE remains a devastating condition.

Intraventricular Interferon a infusions were associated with serious side | effects in our patient and led to treatment discontinuation.

$>$ Prevention through vaccination is the only way to eradicate this fatal complication.. 